\title{
Acoustic emission during quench training of superconducting accelerator magnets
}

\author{
M. Marchevsky ${ }^{\mathrm{a}}{ }^{*}$, G. Sabbi ${ }^{\mathrm{a}}$, H. Bajas ${ }^{\mathrm{b}}$, and S. Gourlay ${ }^{\mathrm{a}}$ \\ ${ }^{a}$ Lawrence Berkeley National Laboratory, Berkeley CA 94720, USA \\ ${ }^{\mathrm{b}}$ European Organization for Nuclear Research (CERN), Geneva, Switzerland
}

\begin{abstract}
Acoustic emission (AE) sensing is a viable tool for superconducting magnet diagnostics. Using in-house developed cryogenic amplified piezoelectric sensors, we conducted AE studies during quench training of the US LARP's high-field quadrupole HQ02 and the LBNL's high-field dipole HD3. For both magnets, AE bursts were observed, with spike amplitude and frequency increasing towards the quench current during current up-ramps. In the HQ02, the AE onset upon current ramping is distinct and exhibits a clear memory of the previously-reached quench current (Kaiser effect). On the other hand, in the HD3 magnet the AE amplitude begins to increase well before the previously-reached quench current (felicity effect), suggesting an ongoing progressive mechanical motion in the coils. A clear difference in the AE signature exists between the untrained and trained mechanical states in HD3. Time intervals between the AE signals detected at the opposite ends of HD3 coils were processed using a combination of narrow-band pass filtering; threshold crossing and correlation algorithms, and the spatial distributions of AE sources and the mechanical energy release were calculated. Both distributions appear to be consistent with the quench location distribution. Energy statistics of the AE spikes exhibits a power-law scaling typical for the self-organized critical state.
\end{abstract}

Keywords: piezoelectric transducers, cryogenic electronics, acoustic emission, quench detection, superconducting accelerator magnets.

\section{Introduction}

Superconducting accelerator magnets are engineered and built with a goal of achieving the design field within a minimal number of quenches. The process of gradual improvement of the spontaneous quench current with every consecutive quench towards reaching a plateau is known as "training" [1-5]. It is a complex phenomenon that involves various types of mechanical motion, micro-crack formation and structural adjustments caused by the Lorentz forces and the associated strain. Lengthy training is not considered acceptable for magnet production and operation in particle accelerators. Minimizing it is considered critical when choosing the best-suited magnet technology for the large accelerator projects, such as the high-luminosity LHC. In order to understand training and properly address it with improvements in magnet design, an adequate set of quench diagnostics and analysis techniques is required. However, despite a long history of accelerator magnet development, such techniques remain limited. In a typical accelerator magnet access for various quench probes is severely constrained by the tight structural packing; the probes may also interfere with the strict requirements for the mechanical and electrical magnet integrity. Strain gauges attached to coils and magnet structural elements, pickup-up loop arrays (quench antennas) in the magnet bore, and voltages from coil segments are typically employed for quench studies. Spatial resolution of all these probes is generally limited by the sparse separation between sensing elements; this limitation becomes more severe for longer magnets, as progressively larger sensor arrays are required. Another challenge for understanding magnet training comes from the complexity of a typical magnet structure, as multiple elements can simultaneously contribute to the premature quenching.

An ideal diagnostic technique should therefore be able to provide spatial information, yet be non-intrusive - preferably using sensors located outside of the magnet structure. One such promising technique is acoustic emission (AE) diagnostics that has a long history [6-11] and nowadays is used extensively for non-destructive evaluation of mechanical stability in various engineering and manufacturing fields. The first use of $\mathrm{AE}$ for diagnostics of quench origins in current-carrying superconductors was demonstrated in 1981 [6]. The technique was later used for diagnostics of a superconducting solenoid [7], and adopted for quench localization [8]. Attempts were made to relate the amplitude of AE bursts to the energy released in the mechanical events during magnet operation [9]. Among advantages of the AE technique are its speed, insensitivity to magnetic fields, excellent adaptability and portability, noninvasiveness, and low cost of setup and operation.

In the present paper, we first review known origins and mechanisms of $\mathrm{AE}$ in the superconducting accelerator magnets, and discuss our AE instrumentation, calibration and signal analysis tools. Next, we present an investigation in the AEs

*Corresponding author, Tel +1 5104952979

E-mail addresses: mmartchevskii@lbl.gov (M. Marchevsky), glsabbi@lbl.gov (G. Sabbi), hugues.bajas@cern.ch (H. Bajas), sagourlay@lbl.gov (S. Gourlay) 
measured during training of $\mathrm{Nb}_{3} \mathrm{Sn}$ superconducting accelerator magnets recently built and tested by a LBNL and US LARP collaboration. We demonstrate localization of the $\mathrm{AE}$ sources during magnet operation and discuss spatial correlation between AE sources and quench locations in the LBNL's high-field dipole HD3. Energy statistics of the AE events is collected during current ramping to quench and results are discussed in connection with the self-organized criticality model that is universally applicable to a large class of complex physical systems.

\section{AE sources in superconducting magnets}

When a superconducting magnet is energized and brought to a quench, a number of mechanical and electromechanical phenomena are taking place that can result in $\mathrm{AE}[10,11]$. A high-field $\mathrm{Nb}_{3} \mathrm{Sn}$ accelerator magnet is typically pre-loaded at room temperature azimuthally and axially, in order to prevent motion in the coils under the Lorentz forces. Significant (>100 MPa) stresses within the magnet coil windings during assembly, cooldown and energizing lead to local deformations, epoxy cracking and slip-stick motion of the conductor and coil parts. These mechanical events are typically the strongest sources of $\mathrm{AE}$. As magnet training continues, new regions of the coil explore progressively larger force variations. The acoustic activity is expected to reflect this process. Apart from purely mechanical events, some AE activity also originates from the numerous flux jumps occurring in the $\mathrm{Nb}_{3} \mathrm{Sn}$ conductor when the magnet is energized, typically at $\sim 10-20 \%$ of the operational current. Flux jumps cause transient local redistribution of the current path in the cable at a sub-millisecond timescale. In a background of a strong magnetic field, these current excursions translate into perturbations of the local Lorentz force and hence produce stress waves in the material propagating away from the source with a speed of sound. This mechanism of AE is similar to the one used in electromagnetic ultrasonic transducers (EMATSs) to generate high-frequency ultrasound [12]. The flux jump induced AE bursts are typically smaller in amplitude than those induced mechanically, and also shifted to higher frequencies [13]. Finally, the most prominent AE event occurs upon quenching of the superconducting magnet. In this case, a sudden formation of a hot spot within the cable generates a volume of locallyelevated stress that subsequently relaxes via acoustic wave generation, followed by a "shake-up" of the entire structure upon current extraction.

Micro-cracks, conductor slip-stick motion and flux jumps are normally local in nature, and therefore can be treated as AE "point sources" for analysis purposes. Stress waves emitted by such point sources propagate within the magnet and become partially converted into the resonant vibrational modes of the magnet structure. Those vibrations can persist over a much longer period of time than the duration of the initial stress wave burst, due to relatively high $(\sim 100)$ mechanical Qfactor of the magnet structure at low temperatures [13]. The background mechanical vibrations may complicate instrumental separation of the acoustic events that are closely-spaced in time. There also exist other generic sources of background noise associated with the cryogen boiling and the magnet test facility operations. Those disturbances, however, are not localized and typically external to the magnet. Therefore AE source localization techniques can be effective for separating the useful signals. In reality, external noises are not too significant, as high mechanical Q-factor of the magnet impedes acoustic energy exchange with the environment.

\section{Instrumentation}

The main components of the AE detection system are amplified piezoelectric sensors, fast DAQ and post-processing software. We use ring-shaped transducers made of SM118 (PZT-8) type piezo-ceramics (Steminc, Inc.). The transducer dimensions are $10 \mathrm{~mm}$ outer diameter, $5 \mathrm{~mm}$ inner diameter and $2 \mathrm{~mm}$ thickness. The transducer is integrated mechanically and electrically with a preamplifier board (see Fig 1a), and the entire assembly is installed on a magnet using a single aluminum set screw passing through the center of the transducer. The preamplifier (gain 3-5) compensates for the reduction of the piezo-transducer sensitivity upon cooling, and converts the high impedance of the bare sensor down to several $\mathrm{k} \Omega$, thus significantly reducing electrical background noise at the ADC input. It is based on a single $p$-channel enhancement-mode GaAs MOSFET allowing operation in a wide range of temperatures from ambient down to liquid helium temperature [14]. Cryogenic operation of the transducer/preamplifier assemblies was verified using a "stick" cryogenic insert to the transport helium Dewar, prior to installation on the magnet. The preamplifier is interfaced to the ADC through an ambient-temperature battery-powered coupling box that electrically isolates the amplifier from the magnet structure and allows use of a single twisted pair of wires for simultaneous cryogenic powering and signal transmission. The electrical schematics of the preamplifier and the interface box are shown in Fig. 1b, the preamplifier frequency response dependence measured at ambient temperature is shown in Fig 1c. The signals were digitized using Yokogawa WE900 DAQ unit equipped with two synchronous double-channel type $7275 \mathrm{ADC}$ cards operating at $1 \mathrm{MHz}$ and four synchronous eight-channel type 7273 ADC cards operating at $100 \mathrm{kHz}$. The sensors were installed at the outer surfaces of magnet structural parts having a direct mechanical contact with the coils. In the LARP quadrupole HQ02b such surface was the bottom load plate (Fig. 2). 

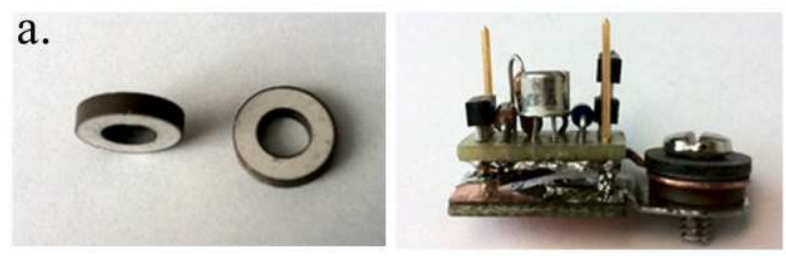

b.

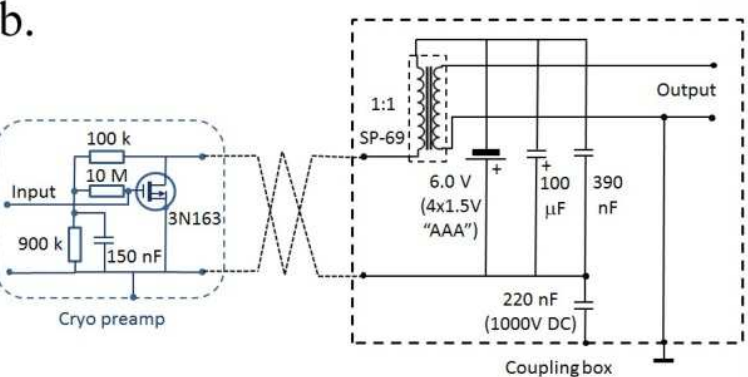

c.

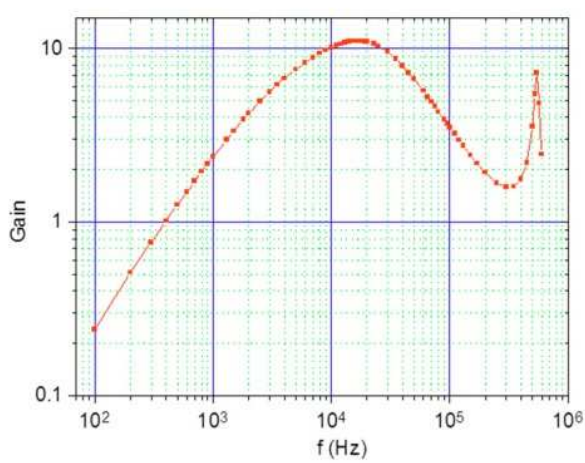

Fig. 1. a) (left) Ring piezoelectric element used as sensing element; (right) an assembled sensor with integrated cryogenic preamplifier. b) Electrical schematics of the cryogenic preamplifier and the coupling box. c) Frequency response curve of the MOSFET preamplifier (measured at ambient temperature.)
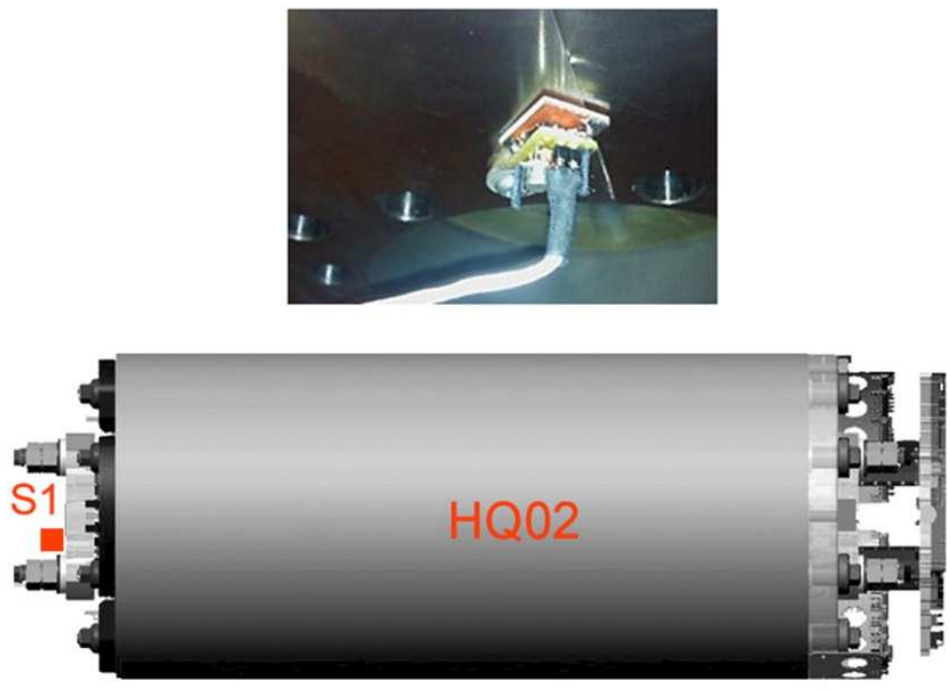

Fig. 2. Photo and sketch of the AE sensor (S1) installed at the load plate (return end, RE) of the quadrupole magnet HQ02. 

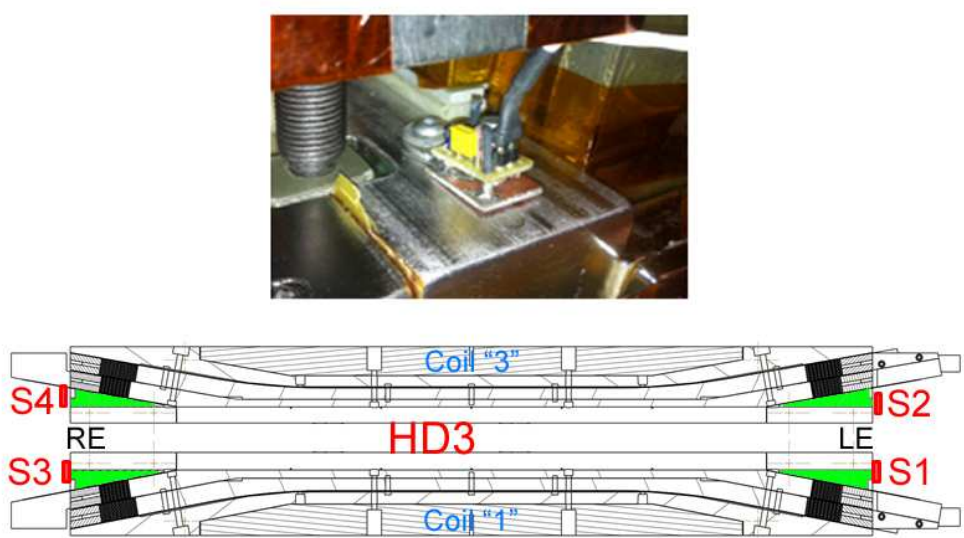

Fig. 3.AE sensors S1, S2 installed at the coil wedges at the return end (RE) and sensors S3, S4 installed lead end (LE) of the high-field dipole HD3b (shown in cross-section). Photo of the sensor attached to the coil wedge is shown at the left.

For the dipole HD3b, the sensors were installed at the stainless steel wedges supporting the flared coil ends (Fig. 3). In the latter case, the distance between the sensor pairs at the opposite coil ends is $1.075 \mathrm{~m}$ and between the sensors at the same magnet end is $0.115 \mathrm{~m}$.

\section{Signal processing and calibration}

\subsection{Signal processing}

Sensor signals are processed in various ways, to analyze AE power, spectral characteristics and source locations. Due to a large size of the acquired data, the data files are split into sets of 0.5-2 s duration and processed sequentially. For source localization, the signals are initially band-pass filtered (typically around $30-50 \mathrm{kHz}$, with $3-5 \mathrm{kHz}$ bandwidth). Filtering improves accuracy of measuring the wave front arrival time, as, due to dispersion in the material, sound waves of different frequency arrive to the sensor at different times. Next, the r.m.s. of the signal is averaged over the set duration and the resulting value is applied as the amplitude normalization factor to that set prior to further processing. In the following step, a detection threshold is set (typically at $\sim 2-3$ times the noise r.m.s. value) and all threshold crossing points are found. They are then sorted to select only those that are preceded by a "silence" (i.e. no threshold crossings) interval of a pre-set duration (typically 2-20 ms). This procedure allows for selecting only the "genuine" time of the first threshold crossing upon wave arrival to the sensor and eliminating all the ringing that follows afterwards. This approach also eliminates detecting any events occurring after the first detection within the pre-defined "silence" interval.

The processing as described is performed for the signals of two sensors of interest (typically those installed at the opposite ends of the coil). Then, the threshold crossing times for each sensor signal are matched, and those that fall within the time interval of $\sim \mathrm{L} / \mathrm{v}_{\mathrm{c}}$ from each other are combined into pairs. Every such resulting pair refers to a genuine AE emission event and the difference between pair elements multiplied by $0.5 v_{c}$ (where $v_{c}$ is the speed of sound, as further discussed in section 4.3 gives a distance from the $\mathrm{AE}$ source to the middle point of the magnet.

\subsection{Energy calibration}

The initial calibration of the system was done at room temperature. We employed a technique similar to that described in [15] where a known test mass is dropped on the magnet structure and a corresponding response is measured with an $\mathrm{AE}$ sensor. When a test object (metal rod or ball) hits the magnet surface, a strain wave is produced and propagates within the magnet structure. The relation between the energy transferred to the magnet at the impact $\mathrm{E}_{0}$ and the voltage response of a piezo-sensor installed in the nearest proximity to the drop point can be expressed as $U=g \sqrt{E_{0}}$, where the general calibration factor $g$ accounts for the material stiffness, the sensor piezoelectric coefficient and the preamplifier electrical gain. In our tests, a stainless rod of $12.2 \mathrm{~g}$ mass was dropped inside a plastic tube of larger diameter from a height $\mathrm{h}_{0}=16$ $\mathrm{cm}$ height down to the test surface of the magnet, yielding $\mathrm{E}_{0}=18.4 \mathrm{~mJ}$ of the associated kinetic energy prior to a first impact. The test object would normally bounce several times, each time reaching progressively lower height $\mathrm{h}_{\mathrm{n}}$, until all its energy has been transferred to the magnet structure. Assuming the same portion of energy $E_{n}$ is transferred to the magnet structure at every consecutive bounce, one could expect $E_{n}$ for the $n-t h$ bounce to scale as:

$$
\mathrm{E}_{\mathrm{n}}=\mathrm{E}_{0} \alpha^{\mathrm{n}}
$$

To verify this scaling, we plot experimental $\mathrm{U}^{2}$ in a log scale versus bounce number; only first six impacts were considered 


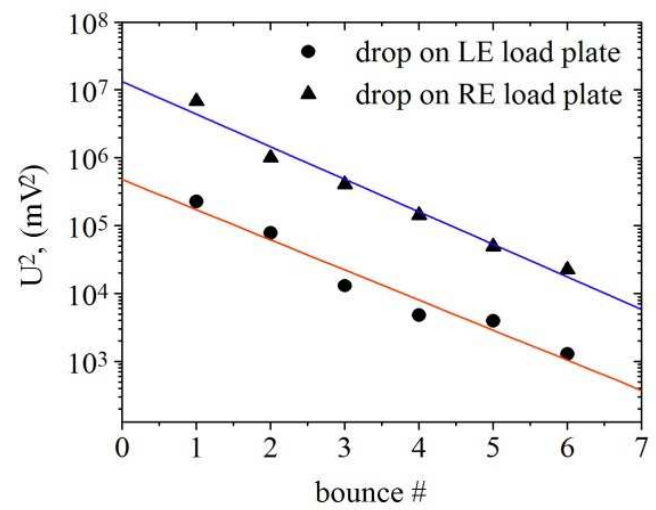

Fig. 4. a) Voltage responses of the AE sensor at the RE load plate to the bounces of the test rod object from the LE and RE load plate. b) Peak amplitudes of $U^{2}$ for each bounce plotted versus the bounce number in a semi-log scale. The energy transfer coefficients are found for each case with linear fitting.

as they were far enough apart in time to allow for a full decay of the magnet structural vibrations after each bounce. The results are shown in Fig. 4; we find $\alpha \sim 0.36$ for the case when mass was dropped on the LE load plate and $\alpha \sim 0.33$ for the RE load plate. Therefore, on average $\sim 65-70 \%$ of the kinetic energy is transferred from the rod to the magnet at each bounce. On the other hand, time interval $\mathrm{t}_{\mathrm{n}}$ between the (n-1)-th and $\mathrm{n}$ - $t h$ bounces is given by $t_{n}=\frac{\sqrt{2 h_{n-1}}}{g}$, and therefore in the ideal case the relation should hold:

$$
E_{n} E_{n-1}=U_{n}^{2} / U_{n-1}^{2}=t_{n-1}^{2} / t_{n}^{2}
$$

The initial fall of the test object from height $\mathrm{h}_{0}$ takes $t_{n}=\frac{\sqrt{2 h_{0}}}{g}=180 \mathrm{~ms}$. Considering just the LE drop experiment, the time measured between the first and the second bounce was $213 \mathrm{~ms}$, and thus free fall duration for height $\mathrm{h}_{1}$ is $\mathrm{t}_{1}=106.5$ ms. Using these numbers, we obtain $E_{1} / E_{0}=t_{0}^{2} / t_{1}^{2} \sim 0.35$ - in good agreement with the previous estimate for the

$\alpha$. However, the time interval between following bounces deviates from one bounce to another for as much as $50 \%$ due to occasional contact between the bouncing rod and the walls of the plastic tube. Based on the resulting average $\alpha \sim 0.36$ and $\mathrm{E}_{0}=18.4 \mathrm{~mJ}$, the energy transferred to the magnet at first impact is $\mathrm{E}_{1} \sim 11.8 \mathrm{~mJ}$. Note that this is an overestimate, as in reality part of the transferred energy will be converted upon impact directly to heat, and so will not contribute to the strain wave. For the RE load plate hosting the sensor, the voltage spike amplitude is $U_{R E}$ drop $=2.61 \mathrm{~V}$ and thus $g \sim 24 \mathrm{~V} / \sqrt{J}$. Given a typical $\sim 1.2 \mathrm{mV}$ p-p ambient noise floor of the amplified $\mathrm{AE}$ sensor, the latter scaling factor translates into the ultimate energy sensitivity of $2.5 \mathrm{~nJ}$ for events occurring in the vicinity of the sensor and at room temperature. Upon cooling down to liquid helium temperature, the piezoelectric coefficient of the sensor ceramic material typically drops $\sim 3-4$ times [16], and thus a corresponding 10-15 times increase in the smallest detectable energy level (up to 25-40 nJ) is expected. The best sensitivity previously reported in the literature for the AE sensors installed on superconducting magnets was $200 \mathrm{~nJ}$ [15]; our system demonstrates at least one order of magnitude improvement in energy sensitivity due to the use of sensor-integrated preamplifiers.

The strain wave excited by the rod drop at RE load plate arrives to the LE attenuated due to energy losses in the material and non-uniformities causing wave scattering. Assuming attenuation of the $\mathrm{AE}$ wave amplitude in the material to follow a universal behavior

$$
A(x)=A_{0} e^{-\beta x}
$$

the attenuation coefficient $\beta$ for the magnet structure can be then estimated as:

$$
\beta=-\left(\frac{1}{L}\right) \ln \left(\frac{U_{\mathrm{LE} \text { drop r.m.s }}}{U_{\mathrm{RE}} \text { drop r.m.s }}\right)
$$

As shape of the strain wave front may change when it propagates through the structure due to dispersion and other factors, we use r.m.s values of the sensor voltage (averaged over $0.2 \mathrm{~s}$ ) rather than the peak values, to improve the accuracy of $\beta$ estimate. We then find $\beta \sim 1.49 \mathrm{~m}^{-1}$.

In HD3, the calibration was done differently, with the magnet mounted vertically at the cryostat header. The sensors S2 and S4 are located in the vicinity of the top load plate and thus their calibration factors can be measured directly by dropping a ball to the top of that load plate. A bronze ball of $3.8 \mathrm{~g}$ weight was dropped from the height of $40 \mathrm{~cm}$, giving 
rise to the initial energy $\mathrm{E}_{0}=14.9 \mathrm{~mJ}$; the energy transfer coefficients was measured as $\alpha_{3}=0.56$ (Coil " 1 ") and $\alpha_{4}=0.5$ (Coil “3”). Then, based on the peak amplitudes of the sensor signals, we obtain $\mathrm{g}_{3}=9.7 \mathrm{~V} / \sqrt{J}$ and $\mathrm{g}_{4}=2.6 \mathrm{~V} / \sqrt{J}$.

For each pair of sensors $\left(\mathrm{S}_{\mathrm{i}}, \mathrm{S}_{\mathrm{j}}\right)$ located symmetrically at the opposite sides of the magnet coil, the ratio $\mathrm{G}_{\mathrm{ij}}=\mathrm{g}_{\mathrm{i}} / \mathrm{g}_{\mathrm{j}}$ can be evaluated experimentally by knocking in the middle of the magnet (on the shell surface) at a point that is symmetric with respect to the sensor locations. For estimation of $\mathrm{G}_{\mathrm{ij}}$ we use r.m.s. values of the sensor voltages averaged over $0.5 \mathrm{~s}$ interval rather than their instant peak values, to improve the accuracy. We find $\mathrm{G}_{31}=3.92$ and $\mathrm{G}_{42}=1.90$. Again, assuming wave amplitude attenuation in the material to follow (3), the voltage response of a sensor separated from the AE source by a distance $\mathrm{x}$ can be expressed as $U_{i}=A_{0} g_{i} e^{-\beta x}$, or $U_{i}=g_{i} \sqrt{E_{A E}} e^{-\beta x}$. For the two sensors $\mathrm{S}_{\mathrm{i}}$ and $\mathrm{S}_{\mathrm{j}}$ separated by the distance $L$, this leads to:

$$
\beta=-\left(\frac{1}{L}\right) \ln \left(G_{i j} \frac{U_{j}}{U_{i}}\right)
$$

Using the ball drop data and the r.m.s. of sensor voltages to calculate $\frac{U_{j}}{U_{i}}$, we find $\beta_{31}=0.45 \mathrm{~m}^{-1}$ for Coil " 1 " and $\beta_{42}=0.55$ $\mathrm{m}^{-1}$ for Coil " 3 ". Finally, for an event of energy $E_{\mathrm{AE}}$ occurring in between the two sensors, the corresponding signals are $\mathrm{U}_{\mathrm{i}}$ $=\mathrm{g}_{\mathrm{i}} \sqrt{E_{A E}} \mathrm{e}^{-\beta \mathrm{x}}$ and $\mathrm{U}_{\mathrm{j}}=\mathrm{g}_{\mathrm{j}} \sqrt{E_{A E}} \mathrm{e}^{-\beta(\mathrm{L}-\mathrm{x})}$, and thus:

$$
E_{A E}=\frac{G_{j i} U_{i} U_{j}}{g_{j}^{2}} e^{\beta_{j i} L}
$$

This expression allows the estimation of $\mathrm{AE}$ event energies occurring anywhere between the two sensors, independently of the event location. The AE power emitted within the coil can be then calculated by integrating the energy given by (6) over a fixed time interval and then normalizing by the integration time. To account for the sensitivity drop of the piezotransducers at cryogenic temperatures, we multiplied raw sensor voltages in (6) by $4 \mathrm{x}$ sensitivity correction factor.

\subsection{Speed of sound}

The speed of sound $v_{c}$ is a crucial parameter for a proper calculation of the AE source location. The complication is that $\mathrm{v}_{\mathrm{c}}$ varies for different modes of propagation and within different materials comprising the magnet structure. The longitudinal (compression) wave is the one that propagates at the highest velocity [17] and hence would be arriving first to the sensor. Longitudinal waves are therefore best suited for precise timing of the AE events. However, in the material they get effectively converted into the shear and surface waves; those waves propagate slower, but ultimately carry most of the energy. The Poisson ratio defines the energy distribution between various propagating elastic wave modes in the given material; in solid structures normally only a small portion of the energy would be carried by the longitudinal wave [17]. Increasing sensitivity and signal to noise ratio of the AE sensor helps to improve temporal resolution when detecting the longitudinal wave arrival.

In practice, the speed of sound can be measured either with the ball dropping experiment as described above, or simply by "knocking" on the magnet structure in the vicinity of one sensor and evaluating the time difference between the signal detected by that sensor and the sensor installed at the opposite end of the magnet. For the HD3 magnet, the ball dropping experiment yielded the time differences of $0.333 \mathrm{~ms}, 0.349 \mathrm{~ms}$ and $0.338 \mathrm{~ms}$ for the three consecutive bounces. Given the distance between the sensors of $1.075 \mathrm{~m}$, we arrive at $v_{c} \sim 3160 \mathrm{~m} / \mathrm{s}$. This value was used for estimation of the AE source locations. It should be noted, that the result for at $v_{c}$ depends on the band-pass filtering parameters (as described in section A), due to dispersion of the acoustic wave in the material. For calibration and data analysis in the rest of the paper, a frequency window centered at $f_{0}=32.5 \mathrm{kHz}$ and of $5 \mathrm{kHz}$ width was applied to the original (non-squared) voltage signals. The accuracy limit of locating AE sources is then $\sim 1 / 2$ of the acoustic wavelength, or $0.5 \lambda_{\mathrm{A}}=\frac{v_{c}}{2 f_{0}} \sim 5 \mathrm{~cm}$.

\section{AE studies during magnet training}

\subsection{Training and $A E$ in the $H Q 02 b$ quadrupole}

$\mathrm{HQ} 02$ is a $\mathrm{Nb}_{3} \mathrm{Sn}$ quadrupole that was developed by LARP in the framework of LHC luminosity upgrade. When the AE experiments were conducted, the magnet had already undergone training up to short sample limit at 4.2 $\mathrm{K}$ [18], and was further trained at $1.9 \mathrm{~K}$ (test $\mathrm{HQ} 02 \mathrm{~b}$ ) [19]. A clear AE precursor to quenching was observed in every current ramp. A detailed study of $\mathrm{AE}$ in $\mathrm{HQ}$ in connection to flux jumps and quench origin localization was earlier reported in [13]. The magnet reached $94 \%$ of short sample at $1.9 \mathrm{~K}$ limit in 10 quenches. The training plot of HQ02b is shown in Fig. 5a and the AE power corresponding to the ramps to quenches 2-6 is shown in Fig. 5b. Intervals between the final currents of the consecutive quenches are shown in the plot with solid bars. The AE bursts first appear at a current value that is close to the 

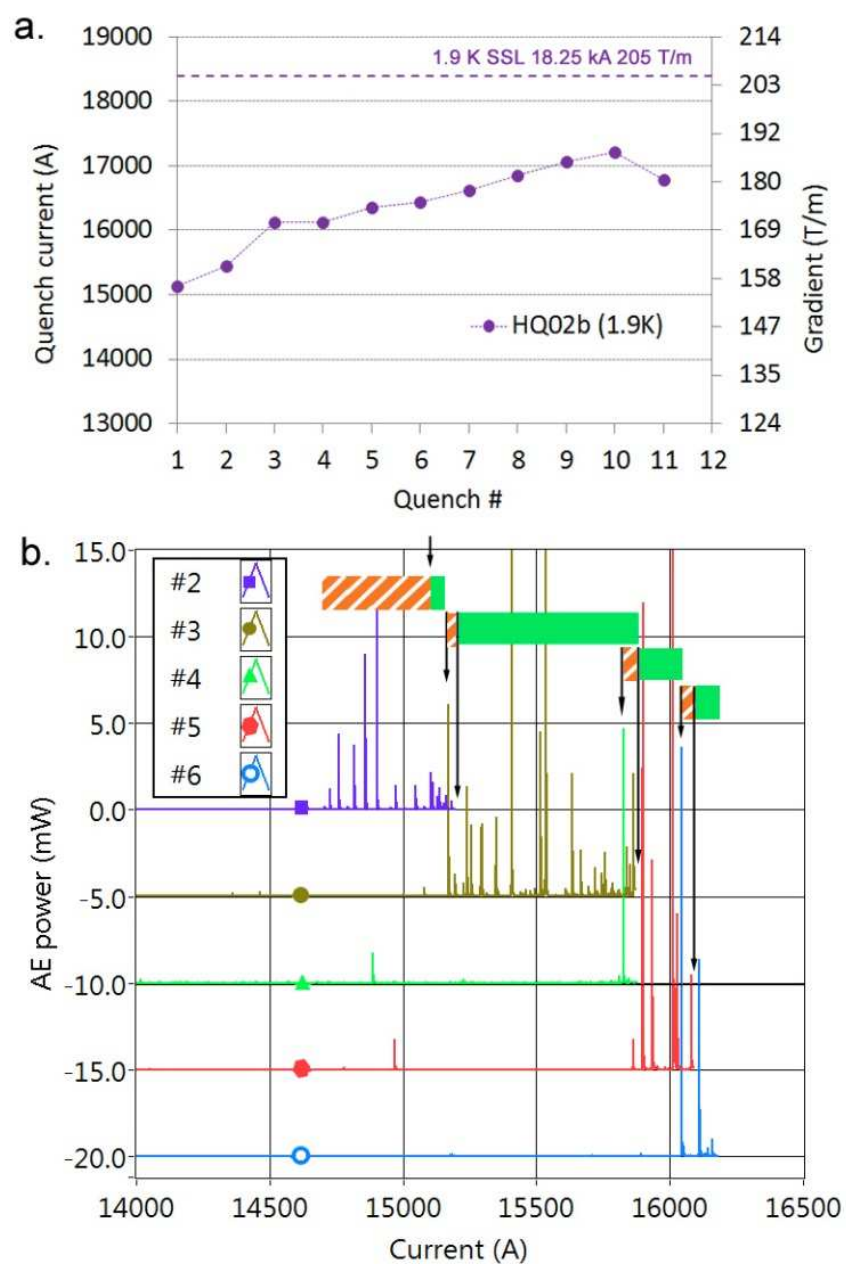

Fig. 5. a. Quench training plot for the HQ02a quadrupole. b. AE power in the consecutive ramps to quench (data acquired at $25 \mathrm{kHz}$ averaged over 20 ms time intervals. Quench current of the first quench (\#1) is 15126 A. Each consecutive graph is offset in y-axis by -5 units for clarity.

previous quench current. This behavior is consistent with the Kaiser effect [20] commonly seen in mechanical structures under repeating load cycles. AE spikes can be attributed to a progressive cracking of epoxy or to a sudden mechanical motion in the coils upon Lorentz force increase above the level that has already been reached in the previous ramp. However, a preliminary comparison with the simultaneously recorded magnet imbalance signal suggests that crack formation is the most likely AE cause. It is because sudden imbalance variations would be typical in case of conductor motion, and no such variations in correlation with the AE events are observed. There is also some overlap between the current values corresponding to the $\mathrm{AE}$ onset and the quench current of the previous run; this is denoted in the plot with hashed bars. This behavior is consistent with the so-called "felicity effect" [21] and normally is attributed to accumulation of damage in the material microstructure or to a progressive mechanical instability under cyclic loading condition of same amplitude. Notably, the interval in the current corresponding to the felicity effect is substantially smaller than the difference between the quench currents of the two consecutive ramps. Therefore, one can conclude that in HQ02 the mechanical support to the coils provided by preload and surrounding structural elements is sufficient to stabilize them under periodic forcing (current ramps) of same magnitude, and to effectively prevent progressive material damage or micro-scale mechanical motion.

\subsection{Training and $A E$ in the $H D 3$ dipole}

$\mathrm{HD} 3$ is a block-type accelerator quality $\mathrm{Nb}_{3} \mathrm{Sn}$ dipole built at LBNL with operational bore fields in the range of 13-15 T [22]. Details of the test (HD3b) were presented in [23]. The magnet exhibited a long training behavior, with $\sim 80$ training quenches completed towards reaching a "plateau" in the quench current at $\sim 87 \%$ of the short sample limit at $4.3 \mathrm{~K}$. The summary of training quenches is shown in Fig. 6a; quenches due to system faults or false detections were omitted from the plot. Acoustic emission measurements were conducted during selected current ramps at 10-100 kSa/s acquisition rates in continuous mode as well as at $1 \mathrm{MSa} / \mathrm{s}$ in triggered mode for a $2 \mathrm{sec}$ interval centered at every quench event. Quench localization using $1 \mathrm{MSa} / \mathrm{s}$ data and details of a typical AE quench precursor in HD3 was earlier presented in [23].

In Fig. 6b raw signals acquired at $10 \mathrm{kSa} / \mathrm{s}$ of the two acoustic sensors (S2 and S4) installed on Coil "3" are shown for 


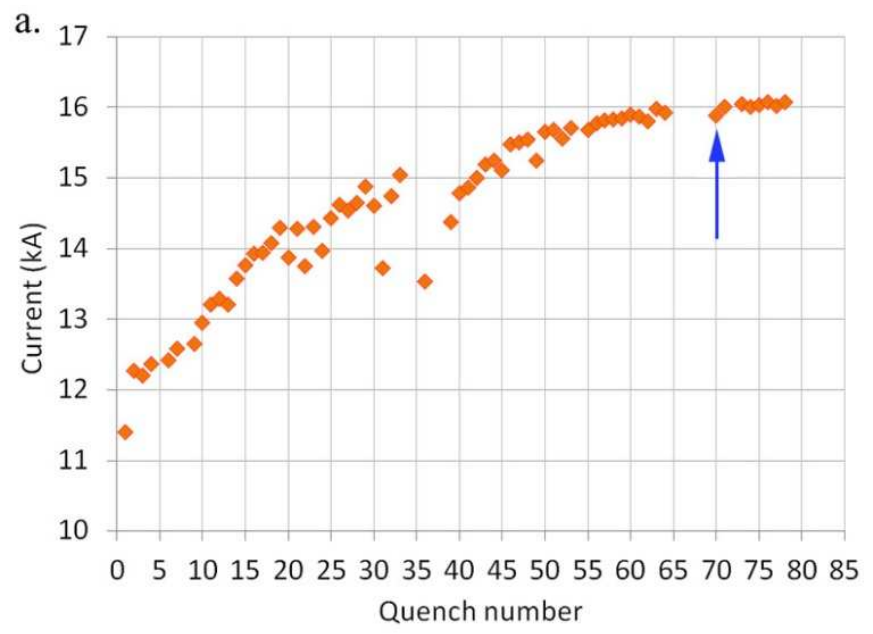

b.
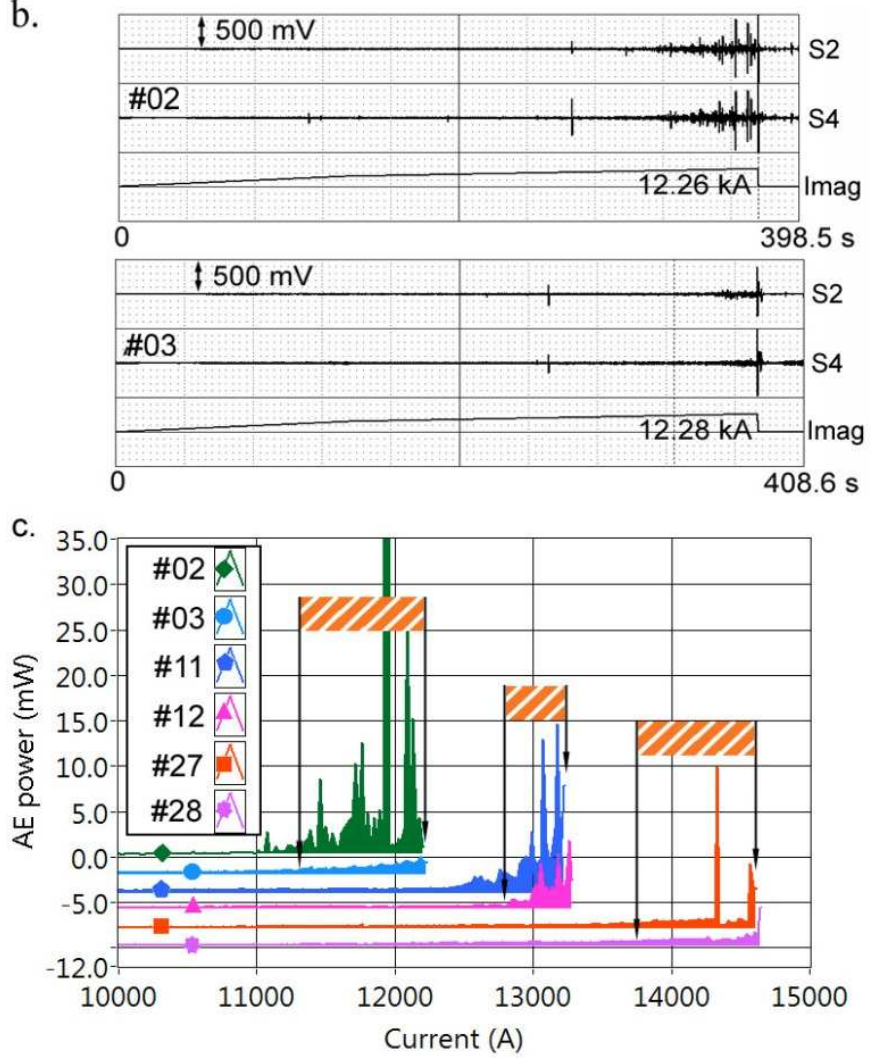

Fig 6. a. Training plot of HD3b. Arrow shows location of the ramp \#70 within the training curve that was used for AE source localization analysis.

b. AE signals acquired at $10 \mathrm{kHz}$ by the sensors 2 and 4 (Coil "3") and the magnet current in the two consecutive quenches at the beginning of the training process (ramps to quench \#02 and \#03). While the quench current values are similar, most of the AE events occur in the ramp \#02 and in the subsequent ramp \#03 the $\mathrm{AE}$ amplitude remains low up to the quench.

c. AE power in Coil "3" during current ramps to quench of HD3b (averaged over $1 \mathrm{~s}$ intervals). Strong AE precursor to quenching is observed, with a progressive shift of the AE onset towards higher currents as training progresses. The rightmost point of each plot corresponds to a quench current. Each consecutive graph is offset by "-5" units in y-axis for clarity.

two consecutive quenches (\#02 and \#03). There is a clear evolution from one quench to another, as large spikes seen prior to quench in A03 change to a more gradually expanding envelope of smaller amplitude spikes. The maximal of the spikes for the sensor S4 reaches $\sim 450 \mathrm{mV}$; using scaling factor $\mathrm{g}_{4}=2.6 \mathrm{~V} / \sqrt{J}$ in combination with the cryogenic sensitivity correction factor $\mathrm{x} 4$, this voltage corresponds to the instant release of the mechanical energy of $\sim 0.5 \mathrm{~J}$. This energy level is quite large compared to the energy required for quenching a short portion of the superconducting cable [5]. We therefore assume that these AEs may be related to epoxy cracking and/or plastic motion events that occur outside of the cable and therefore are not directly converted into heating of the superconductor. In Fig. 6c plots of AE power as function of the 
magnet current are shown. The original sensor data were acquired at a rate of $10 \mathrm{ks} / \mathrm{s}$. The power was calculated using (1); raw sensor voltages were multiplied by $4 \mathrm{x}$ cryogenic sensitivity correction factor integrated over $0.5 \mathrm{~s}$. time intervals, and normalized by the integration time. Results for the six quenches shown represent various stages of the training process In all these quenches the current was ramped up to $7 \mathrm{kA}$ at $50 \mathrm{~A} / \mathrm{s}$ and then the rate was changed to $20 \mathrm{~A} / \mathrm{s}$ for the rest of the ramp. The acoustic emission intensity increases steeply at around $\sim 90 \%$ of the quench current. While the onset of the $\mathrm{AE}$ signal shifts to higher currents as training progresses, it is notable that, unlike in the HQ02 case, there is a significant difference between the current value characteristic to the onset of the $\mathrm{AE}$ and the quench current reached of the previous ramp. In every following quench the spikes appear at a notably lower current level than the quench current reached in the preceding ramp. This is a manifestation of a strong "felicity effect" [21] which dominates the magnet AE behavior. Towards the end of the training process, the quench location map shows a significant clustering at $\sim 15-30 \mathrm{~cm}$ from the center towards both coil ends, being primarily in one of the coils ("Coil 3"); see Fig. 7a. The AE signals evolve to a more gradually expanding envelope of closely-spaced spikes; the large distinct spikes, as in quench \#02, are no longer observed. The signal amplitude increase begins from the low values of current and there is no longer a clear threshold for the onset of AE. An example is shown in Fig. 6b (quench \#70, marked in Fig 6a); the dataset was acquired at a $100 \mathrm{ks} / \mathrm{s}$ rate for the total duration of $582.3 \mathrm{~s}$ ). In order to determine preferential localization of the AE sources in the trained state, we analyze a $500 \mathrm{~s}$ long portion of this dataset, which corresponds to the current range that is still below the quench current, and the training plateau current level. Results are summarized in Fig. 7b. The AE source distribution between sensors S1 and S3

a.

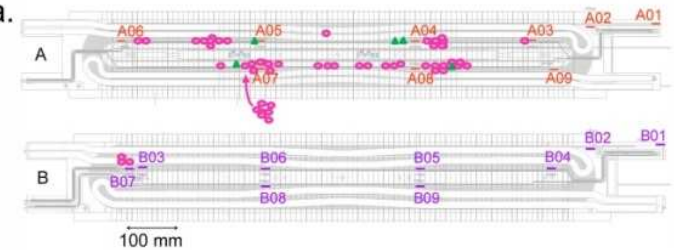

b.

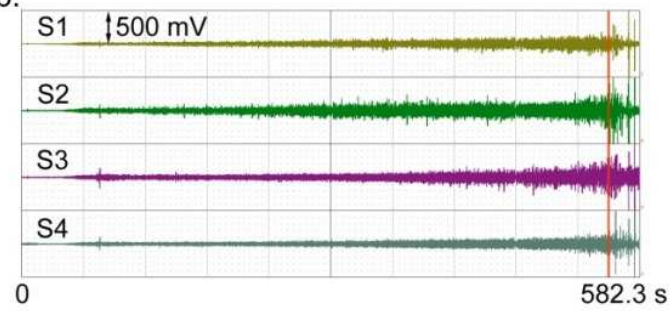

c.
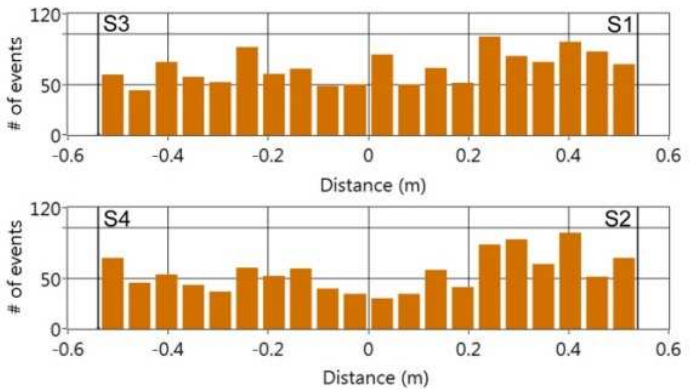

d.
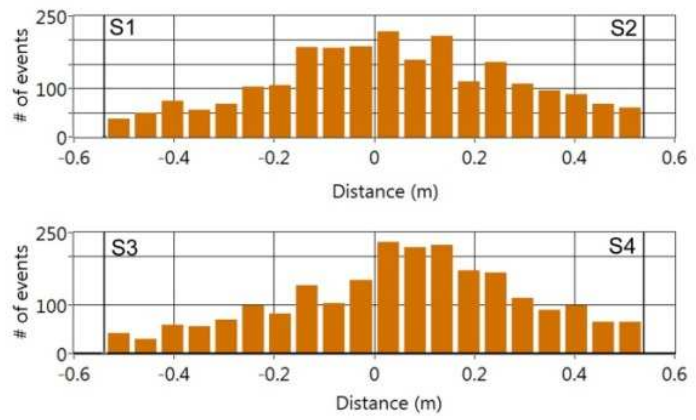

Fig.7. a. Summary of the quench locations in HD3b; circles denote locations for Coil "3"; triangles - for Coil "1" (adopted from [23]).

b. Raw signals of the four AE sensors during ramp to quench \#70 at $15877 \mathrm{~A}$. The time of the quench is marked with a vertical line.

c. Histogram plots of the AE source location distribution along the magnet length found based on AE signals acquired form Coil 1 (S1, S3) and Coil 3

(S2, S4). d. Plots for the magnet lead end (S1, S2) and return end (S3, S4) are shown for comparison. 

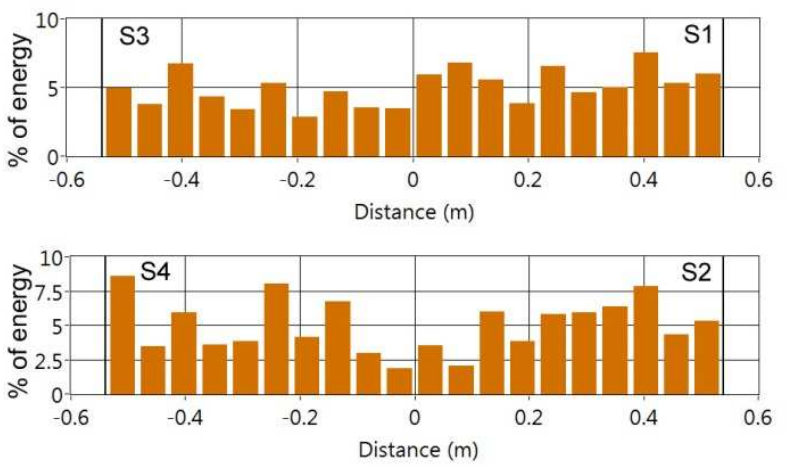

Fig.8. Histogram plots of the net AE energy release distribution along the magnet length in Coil "1" (S1, S3) and Coil "3" (S2, S4) during ramp to quench \#70.

(Coil "1") exhibits several local maxima; the highest is at $~ 24 \mathrm{~cm}$ distance from the center towards the LE. The AE source distribution between sensors S2 and S4 (Coil "3") is "v"-shaped and exhibits broad maxima at 25-40 cm towards LE, and around $20 \mathrm{~cm}$ towards RE (Fig. 7c). At the same time, for the sensor pairs at the same end (LE or RE) of the magnet, $(\mathrm{S} 1, \mathrm{~S} 2)$ and $(\mathrm{S} 3, \mathrm{~S} 4)$, which are separated by $\sim 11.5 \mathrm{~cm}$ distance, the AE signals are expected to arrive simultaneously or with a very small relative delay. Indeed, nearly symmetric distributions with a single maximum around the middle of the magnet are found in both cases (Fig. 7d). Next, AE energy release distribution over the length of the magnet was calculated using (6) for all detected events. The resulting histogram plots are shown in Fig. 8. It is even more evident from these plots, that the energy is released non-uniformly in the coils, with local maxima at $\sim 8 \mathrm{~cm}$ and $\sim 45 \mathrm{~cm}$ toward the LE for Coil "1" and at $16 \mathrm{~cm}$ towards RE and at $40 \mathrm{~cm}$ towards LE for Coil " 3 . It is notable, that in Coil " 3 " the AE source density is less uniform than in Coil "1", given about twice as large variation between the energy minimum and maximum. When comparing with the quench location map of Fig. 7a, these results imply that the sections of the coils that emit most of the $\mathrm{AE}$ energy during current ramp are also those where most of the quench locations are situated. Such correlation is interesting and calls for further studies. If proven to be reproducible in the future tests of other magnets, it could turn the $\mathrm{AE}$ sensing technique into a tool for predicting the most likely spatial distribution of quench regions. The result also suggests that quenching in HD3 may be caused by a mechanical event of same type as those detected with the AE sensors during the current ramp. Similar connection between the friction-induced AE events and coil quenching was made for example in [24]. An estimate for the amount of heat required to raise temperature of the HD3 Rutherford cable from $4.2 \mathrm{~K}$ to $14.2 \mathrm{~K}$ yields approx. $7.7 \mathrm{~mJ}$ per $\mathrm{cm}$ length (assuming the cable material is all copper and taking into account both electronic and phonon heat capacity terms). This number is similar to the mechanical energy released with the pre-quench AE events (such as those shown in Fig. 6c) in $1 \mathrm{~s}$. It is therefore plausible to assume that the AEs are closely connected to the quench. The connection can be further explored by plotting the number of $\mathrm{AE}$ events of amplitude larger than $E_{A E}$ versus $E_{A E}$. The result for Coil "1" (sensors S1 and S3) in the ramp \#72 of HD3b is shown in Fig. 9. In this ramp, the magnet current has reached $15951 \mathrm{~A}$. In a log-log scale, the data points can be well fitted with a straight line, yielding a power-law dependence of $N_{E>E_{A E}}$ versus $E_{A E}$ with an exponent of $\sim 2.2$. The observed power law dependence is same as those found in

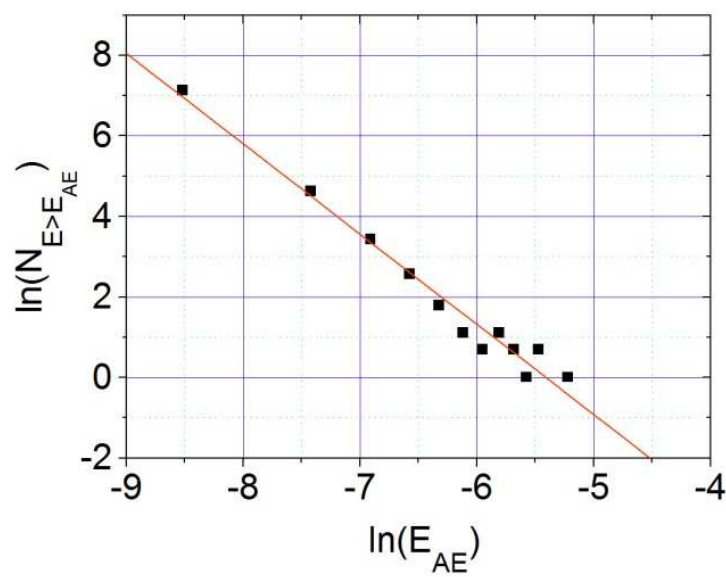

Fig. 9. Cumulative number of the $\mathrm{AE}$ events of energy $E>E_{A E}$ in Coil "1" during ramp \#72 plotted versus $\mathrm{E}_{\mathrm{AE}}$. The linear fit of the data reveals a power-law dependence of $N_{E>E_{A E}}$ upon $E_{A E}$ with an exponent of 2.2. 
the distribution of earthquake sizes (Gutenberg-Richter law), dynamics of sandpiles, fracture in disordered materials, Barkhausen noise in magnetics and in many other systems with a manifestation of the scale-invariant behavior and selforganized criticality (SOC) [25,26]. The essence of the self-organized criticality concept is the existence of a universal physical mechanism reproduced at all energy scales and allowing the system to achieve a critical state without action of the external driving factor. Slip-stick motion [27] and progressive crack formation under increasing loading in heterogeneous materials [28] conform to the SOC model. Here, we may assume that both of these mechanisms, namely epoxy cracking in the coil windings and slipping between the cable and the central coil parts, are responsible for the AEs. Both mechanisms are also ultimately connected to magnet quenching through an associated heat release and conductor motion. It would be therefore interesting to further explore if a variations of the critical exponent or a divergence from the power law behavior can be found in the close vicinity of the quench current. A correlation between the AE statistics and variations of global mechanical response of coils measured by the strain gauges during current ramping is another potential topic to be addressed for further understanding of the present result.

\section{Discussion}

Comparison of the training behavior and the $\mathrm{AE}$ data for the two different magnets reveals a number of important similarities and differences. In the HQ02 quadrupole training, large isolated AE spikes are typically seen just prior to quenching. One observes a clear AE Kaiser effect and minimal felicity effect. On the contrary, in the HD3 dipole evolution from untrained to trained state is reflected in the change from large isolated spikes detected just prior to quenching to a gradual increase in amplitude of the AE noise envelope, starting from the low currents. Based on the difference in instant energy release between these two AE regimes, one can speculate that they manifest epoxy cracking and slip-stick motion, respectively. The HD3 training process is dominated by the felicity effect in AE. Since the latter is normally taken as evidence of mechanical instability or progressive material damage under cyclic load, one can speculate that such phenomena may indeed take place within the HD3 magnet coils. A weaker or less uniform mechanical support by the structural elements in HD3 could be a cause for the ongoing mechanical activity observed in our AE experiments. Given the correlation found between the AE source density and quench locations in HD3, it is plausible to suggest that the felicity effect in this magnet may be connected to its slow training and, possibly, to the observed saturation at $87 \%$ of the short sample limit. However, before making a definitive conclusion regarding such connection, one still needs to eliminate the possibility of cable damage in HD3 as being a potential cause of its performance limitation.

\section{Conclusions}

$\mathrm{AE}$ measurements provide a unique insight into the physics of processes taking place during training and operation of the superconducting accelerator magnets. They allow assessment of the general stability of the magnet's mechanical state with respect to a development of cracks, plastic deformations, and slip-stick motion, and, potentially identify deficiencies in coil mechanical support. Furthermore, the data suggest a connection between magnet's AE signature and its training behavior. In the trained state, the coil winding regions that are more prone to quenching also appear to produce most of the AE power during current ramping. This correlation is worth further exploration as a potential tool for a quick assessment of a quench probability distribution in the windings. As a magnet is being energized, a general scaling of the AE event count with the event energy is typical for a system with self-organized criticality. Correlating variation of the scaling exponent with the strain measurements may be an interesting topic for future studies. Further development of the AE sensing technique aimed at understanding micro-scale physics of the superconducting magnets will benefit our understanding of quench training and help optimizing magnet design for the ultimate quench performance.

\section{Acknowledgements}

The authors are thankful to M. Turqueti and R. Albright for assistance with the sensor instrumentation development and magnet testing, J. Feuvrier and M. Bajko for assistance during the HQ02b test, and to X. Wang, P. Bish, D. Cheng, T. Lipton for technical assistance during the HD3b test. Authors are also thankful to S. Prestemon, V. I. Datskov and A. McInturff for useful discussions.

This work was supported by the U.S. Department of Energy under Contract No. DE-AC02-05CH11231 and the US LHC Accelerator Research Program (LARP). Part of this work was supported by the European Commission FP7 HiLumi LHC Grant Agreement 284404. 


\section{References}

[1] G. Pasztor and C. Schmidt, "Dynamic stress effects in technical superconductors and the "training" problem of superconducting magnets", J. Appl. Phys. 49, 886 (1978)

[2] H. Brechna and P. Turowski, "Training and degradation phenomena in superconducting magnets," Proc. 6th Intl. Conf. Magnet Tech. (MT6) (ALFA, Bratislava, Czechoslovakia) 597, (1978).

[3] A. V. Tollestrup, "Care and training of superconducting magnets", IEEE Trans. Magn., vol. MAG-17, no. 1, pp. 863-872, Jan. 1981.

[4] P. P. Granieri and E. Todesco, "Slip-stick mechanism in training the superconducting magnets in the large hadron collider", IEEE Trans. Appl. Supercond. 21, pp. 3555-3560 (2011).

[5] P. Ferracin and S. Caspi, "Finite element model of training in the superconducting quadrupole magnet SQ02, Cryogenics, 47, 11-12, pp. 595-606, (2007) DOI: 10.1016/j.cryogenics.2007.08.006.

[6] O. Tsukamoto, J. F. Maguire, E.S. Bobrov, and Y. Iwasa, "Identification of quench origins in a superconductor with acoustic emission and voltage measurements", Appl. Phys. Lett. 39, 172 (1981)

[7] "Discussion on acoustic emission of a superconducting solenoid", M. Pappe, IEEE Trans. on Magn., 19, 1086 (1983)

[8] O. Tsukamoto and Y. Iwasa, "Acoustic emission triangulation of disturbances and quenches in a superconductor and a superconducting magnet", Appl. Phys. Lett. 40, 538 (1983)

[9] O. O. Ige, A. D. Mclnturf and Y. Iwasa, "Acoustic emission monitoring results from a Fermi dipole", Cryogenics 26, pp. 131-151 (1986)

[10] O. Tsukamoto and Y. Iwasa, "Sources of acoustic emission in superconducting magnets", J. Appl. Phys. 54, 997 (1983).

[11] Y. Iwasa, "Mechanical Disturbances in Superconducting Magnets-A Review", IEEE Trans on Magn., 28, 113 (1992)

[12] R.B. Thompson, "Physical Principles of Measurements with EMAT Transducers", Ultrasonic Measurement Methods, Physical Acoustics Vol. XIX, by. Edited by R.N. Thurston and Allan D. Pierce, Academic Press, 1990

[13] M. Marchevsky, X. Wang, G. Sabbi and S. Prestemon, "Detecting mechanical vibrations in superconducting magnets for quench diagnostics", Proceedings of the WAMSDO 2013 Workshop, ed. by E. Todesco, CERN, Geneva 2013, DOI: 10.5170/CERN-2013-006

[14] H.-P. Rust, M. Heyde, and H.-J. Freund, "Signal electronics for an atomic force microscope equipped with a double quartz tuning fork sensor", Rev. Sci. Instr. 77, 043710, (2006)

[15] O. Tsukamoto, Y. Iwasa, "Acoustic Emission Diagnostic \& Monitoring Techniques for Superconducting Magnets", Adv. Cryogen. Eng. 31, pp 259268 (1986)

[16] Cady, W.G. "Piezoelectricty", Vol. 1 \& 2. Dover Publications, NY (1964)

[17] "Dynamic Behavior of Materials" by M. A. Meyers, Wiley-Interscience (1994)

[18] G. Chlachidze, G. Ambrosio, M. Anerella, F. Borgnolutti, R. Bossert, S. Caspi, D. W. Cheng, D. Dietderich, H. Felice, P. Ferracin, A. Ghosh, A. Godeke, A. R. Hafalia, M. Marchevsky, D. Orris, P. K. Roy, G. Sabbi, T. Salmi, J. Schmalzle, C. Sylvester, M. Tartaglia, J. Tompkins, P. Wanderer,

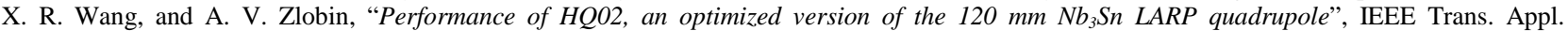
Supercond., vol. 24, no.3, 4003805, June 2014, DOI: 10.1109/TASC.2013.2285885

[19] H. Bajas, G. Ambrosio, M. Anerella, M. Bajko, R. Bossert, L. Bottura, S. Caspi, D. Cheng, A. Chiuchiolo, G. Chlachidze, D. Dietderich, H. Felice, P. Ferracin, J. Feuvrier, A. Ghosh, C. Giloux, A. Godeke, A. R. Hafalia, M. Marchevsky, E. Ravaioli, G. L. Sabbi, T. Salmi, J. Schmalzle, E. Todesco, P. Wanderer, X. Wang, M. Yu, "Cold Test Results of the LARP HQ02-b magnet at 1.9 K", IEEE Trans. Appl. Supercond., vol. 25, no 3, 4003306, June 2015, DOI: 10.1109/TASC.2014.2378375

[20] "Erkenntnisse und Folgerungen aus der Messung von Geräuschen bei Zugbeanspruchung von metallischen Werkstoffen", J. Kaiser, Archiv Eisenhüttenwesen, $241 / 2$ pp. 43-45 (1953)

[21] T. J. Fowler, "Acoustic Emission Testing of Fiber Reinforced Plastics" Proc. Paper 15021, J. Tech. Councils, ASCE, 105 (TC2), pp. 281-289 (1979)

[22] D. W. Cheng, S. Caspi, D. R. Dietderich, H. Felice, P. Ferracin, A. R. Hafalia, M. Marchevsky, S. Prestemon, and G. Sabbi, "Design and Fabrication Experience With $\mathrm{Nb}_{3} \mathrm{Sn}$ Block-Type Coils for High Field Accelerator Dipoles", IEEE Trans. Appl. Supercond. 23, 4002504, June 2013, DOI: 10.1109/TASC.2013.2246811

[23] M. Marchevsky, S. Caspi, D. W. Cheng, D. R. Dietderich, J. DiMarco, H. Felice, P. Ferracin, A. Godeke, A. R. Hafalia, J. Joseph, J. Lizarazo, P. K. Roy, G. Sabbi, T. Salmi, M. Turqueti, X. Wang, S. Prestemon, "Test of the High-Field Nb ${ }_{3}$ Sn Dipole Magnet HD3b", IEEE Trans. Appl. Supercond. 24, 4002106, (2014) DOI: 10.1109/TASC.2013.2285881

[24] K. Seo, S. Nishijima, K. Takahata, and T. Okada, "Detection and evaluation of instability in race-trackshaped superconducting coils", Proceedings of 11th International Conference on Magnet Technology (MT-11), vol. 2, pp. 1207-1212, Elsevier Sci. Pub., NY (1990).

[25] J. P. Sethna, K. A. Dahmen, and C. R. Myers, "Crackling noise", Nature 410, pp 242-250 (2001)

[26] D. Markovića, C. Grosa, "Power laws and self-organized criticality in theory and nature", Phys. Reports 536, pp 41-74, (2014), DOI: 10.1016/j.physrep.2013.11.002

[27] H. J. S. Feder and J. Feder, "Self-organized criticality in a stick-slip process", Phys. Rev. Lett. 66, 2669 (1991), DOI:10.1103/PhysRevLett.66.2669

[28] G. Pontuale, F. Colaiori, and A. Petri, "Slow crack propagation through a disordered medium: Critical transition and dissipation", Euro Phys. Lett. 101, 16005 (2013), DOI: 10.1209/0295-5075/101/16005 